University of Nebraska - Lincoln

DigitalCommons@University of Nebraska - Lincoln

Public Health Resources

Public Health Resources

2005

Measurements of Constraint of Total Knee Replacement

H. Haider

University of Nebraska, Omaha, USA

P.S. Walker

New York University, Hospital for Joint Diseases, Veterans Administration Hospital, Annex Building A 423, East 23rd Street, New York, NY

Follow this and additional works at: https://digitalcommons.unl.edu/publichealthresources

Part of the Public Health Commons

Haider, H. and Walker, P.S., "Measurements of Constraint of Total Knee Replacement" (2005). Public Health Resources. 43.

https://digitalcommons.unl.edu/publichealthresources/43

This Article is brought to you for free and open access by the Public Health Resources at DigitalCommons@University of Nebraska - Lincoln. It has been accepted for inclusion in Public Health Resources by an authorized administrator of DigitalCommons@University of Nebraska - Lincoln. 


\title{
Measurements of constraint of total knee replacement
}

\author{
H. Haider ${ }^{\mathrm{a}}$, P.S. Walker ${ }^{\mathrm{b}, *}$ \\ ${ }^{a}$ Department of Orthopaedic Surgery, University of Nebraska, Omaha, USA \\ ${ }^{\mathrm{b}}$ Orthopaedic Department, New York University, Hospital for Joint Diseases, Veterans Administration Hospital, Annex Building A 423, \\ East 23rd Street, New York, NY 10022, USA
}

\begin{abstract}
Measurement of the constraint of total knee components in a test machine provides an objective method of describing the laxity and stability characteristics of the implant itself, independent of the knee joint into which it would be implanted. A special fixture was designed and fitted to a Bionix multi-channel loading machine. The test consisted of applying a compressive load, applying a cyclic AP force or internal-external torque, and measuring all of the displacements and rotations. Three different commonly-used TKR's showed widely different constraint characteristics. In the cyclic AP test, along with the cyclic AP displacement, displacements and rotations occurred in the other directions. This indicated that all degrees of freedom should be free to move, otherwise anomalous results would be obtained. The paper concludes with recommendations for standardized constraint tests.
\end{abstract}

Keywords: Total knee replacement (TKR); TKR constraint; TKR laxity; TKR stability; TKR kinematics; TKR testing

\section{Introduction}

Constraint is a term which describes the resistance to displacements and rotations between the femoral and tibial components of a total knee when forces and moments are applied. Previous methods for defining and measuring constraint have been reported (Black and Greenwald, 1979; Black et al., 1977; Heim et al., 2001; Luger et al., 1997; Thatcher et al., 1987; Werner et al., 1978). Typically, the components have been held in fixtures and an axial compressive force has been applied. An anterior-posterior (AP) shear force has then been applied and the displacement to a defined shear force value, or the displacement at dislocation has been measured. Force-displacement and torque-rotation curves have been used to describe the behaviour and to define stiffness at particular locations on the curves. A hysteresis loop has invariably occurred which has been attributed to friction (Luger et al., 1997). In the tests, degrees of freedom of the components other than those being tested were not provided for, because most designs were symmetric and the fixtures would become

*Corresponding author. Department of Orthopaedic Surgery, New York University, Veterans Administration, Annex Building 2, 423 East

23 Street, New York, NY 10010, USA.

E-mail address: ptrswlkr@aol.com (P.S. Walker). more complicated. The numerous possible test conditions and definitions, and design configurations, have confounded the attempt to define constraint simply. In our study, we carried out tests on contemporary designs using fixtures providing multiple degrees of freedom, to identify constraint characteristics from which to determine recommendations for testing unlinked condylar replacement designs, or linked knees with an intercondylar stabilizing mechanisms where varus-valgus constraint comes into play. Flexion-extension constraint is effectively zero except in the case of linked designs which have limits at the extremes of motion.

These different constraints can be considered independently with respect to the geometry of the implant. For example, in a condylar design, as the sagittal radius of the plastic changes from infinite (flat) to small (approaching that of the femoral radius), the constraint would progress from zero to a high value. For a given tibial sagittal radius, an increasing femoral sagittal radius would increase the constraint. Rotational constraints depends upon the radii of curvature in both the sagittal and frontal planes. Both modes of constraint will be affected by geometrical features of the tibial surface, such as arcuate tracks, varying radii of curvatures, or flat regions. Asymmetry of the femoral or tibial surfaces can have a major effect. The friction between the bearing surfaces adds to the constraint. 
Deformation of the plastic will in general reduce the constraint and can add a time-dependent factor. Finally, over the long-term, or on repetitive testing, permanent deformation or wear will affect the constraint.

It can be seen that these numerous factors make an analytical approach to the characterization of constraint, although possible, difficult due to the variable geometrical and material properties involved. It is the purpose of this paper to present the rationale for an experimental approach, one which can readily be carried out in biomechanical laboratories using available equipment with the addition of suitable jigs and fixtures.

\section{Methodology}

\subsection{Definitions}

At this stage, a simple definition for constraint will be proposed: constraint is described by a force-displacement curve, or by a torque-rotation curve, together with the magnitude of the compressive force applied across the bearing surfaces. Consider a TKR placed in a neutral position, with a compressive force applied. A shear force or torque is then applied, and the displacement or rotation is then measured. Constraint is expressed as the force per unit compressive force, per unit displacement for the total displacement range

$C=1 / W \times(F / a)$,

where $C$ is the Constraint $\left(\mathrm{L}^{-1}\right), F$ the applied force at a defined displacement from the neutral position, $a$ the magnitude of the displacement, $W$ the applied axial compressive force.

An alternate definition will be advanced based on the slope of the force-displacement curve at a selected shear force or displacement value:

$C=1 / W \times \mathrm{d} F / \mathrm{d} a$.

Hence, in order to quantify constraint, suitable equipment and a test methodology is required to obtain the force-displacement curves.

\subsection{Test equipment}

In order to investigate the constraint characteristics of different types of TKR as a basis for proposing a universally applicable method for defining and measuring constraint, a mechanical test machine was used together with specially constructed fixtures. A schematic is shown in Fig. 1. The machine (MTS Bionix) was capable of applying and controlling an axial compressive force, an axial torque, and a force perpendicular to the vertical axis of the machine (e.g. AP force).

The femoral component was attached to the upper shaft such that it could be moved along the vertical axis.

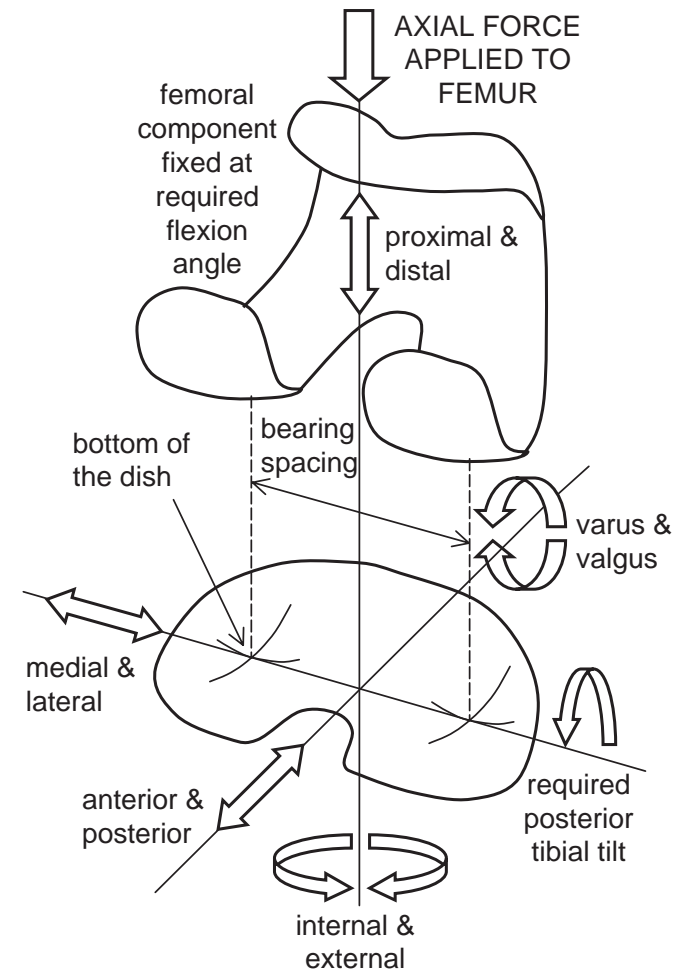

Fig. 1. Schematic representation of the test equipment. All of the displacements and rotations indicated were measured during the tests. Axial force or torque were applied to the femur. AP force was applied to the tibia. The femur (set at the required flexion) could only move vertically. Due to the fixture, the tibia (set at the required posterior slope) was free to move AP, ML, rotation, and varus-valgus.

The shaft applied the vertical force and the torque. The femoral component was mounted at the required angle of flexion with a medial-lateral (ML) line across the lowest points on the lateral and medial condyles intersecting the vertical axis.

The tibial component was mounted on a platform which was fitted with linear bearings to allow unrestrained motion in the AP and ML direction. Special low friction slides were used. Testing under numerous loading conditions up to $6 \mathrm{kN}$, and with the slides at different locations, gave friction forces less than $3 \mathrm{~N}$. In the fixture, the tibial component was allowed unrestrained varus-valgus rotation about an AP axis through the joint line. The machine allowed dynamic data logging of the following variables: axial force and displacement, AP force and displacement, axial torque and rotation angle, ML displacement, and varus-valgus angle. Time was the phase synchronizing variable.

\subsection{General test method}

The following method was applied to all of the tests, whether AP or internal-external rotation. The femoral component was mounted in the actuator at the required flexion angle, centered in the AP and ML directions. The tibial component was mounted horizontally or with a 
posterior tibial tilt as specified in the surgical technique. The tibial fixture was attached to the horizontal force actuator for all tests. During set-up, the component was centered, and the ML displacement and varus-valgus measurements were zeroed.

The first step was to determine the neutral position of the tibial component in an AP direction specified as when the femur was at the 'bottom of the tibial dish'. Distilled water was applied to the tibial surfaces. A $100 \mathrm{~N}$ axial compressive force was applied through the femoral actuator with the femoral component being visually located on the tibial component at approximately the neutral position. Using the AP force actuator, the tibial was moved horizontally, observing the plot of the vertical versus horizontal displacement on the computer screen. The lowest point of this curve was clearly seen and designated as the neutral position in the control software. Horizontal force versus horizontal displacement gave the same result and could equally have been used. The instruments and scales for AP force, AP displacement, and vertical displacement, were all set to zero at this neutral position.

The next step was to determine the limits of AP displacement over which the test would be carried out. Distilled water was again applied to the tibial surface. The rotational control of the femur was set to maintain zero axial torque. An axial compressive force of $712 \mathrm{~N}$ (1 BW) was applied. The AP tibial actuator was manually controlled to move the tibial component anteriorly. The plot of AP force versus AP displacement was continuously observed. When the slope of the curve became negative (Fig. 2), that was defined as the anterior limit. The same procedure was used to define the posterior limit. These limits were then programmed into the control software.

Calf serum was then applied to the bearing surfaces. The actual test was then carried out under AP displacement control, ramping the displacement between the anterior and posterior limits through five full cycles. The first two cycles were not recorded, while the next three cycles were recorded and the data stored. The data were recorded of the axial force (nominally $712 \mathrm{~N}$ ), the AP force, the AP displacement, the vertical displacement, the rotational torque (nominally zero), the rotational angle, ML displacement and varus-valgus rotation. After a lapse of 2-3 min, a second set of five cycles was recorded, followed by yet a third set. These repetitions were to study repeatability.

The entire procedure as for AP displacement was then repeated for internal-external rotation. This time, the angle-control rotation was applied through the femur, while the AP actuator was controlled to maintain zero AP force and the same $712 \mathrm{~N}$ controlled axial compressive force.

Additional tests were also carried out to determine the effect of controlling certain degrees of freedom in

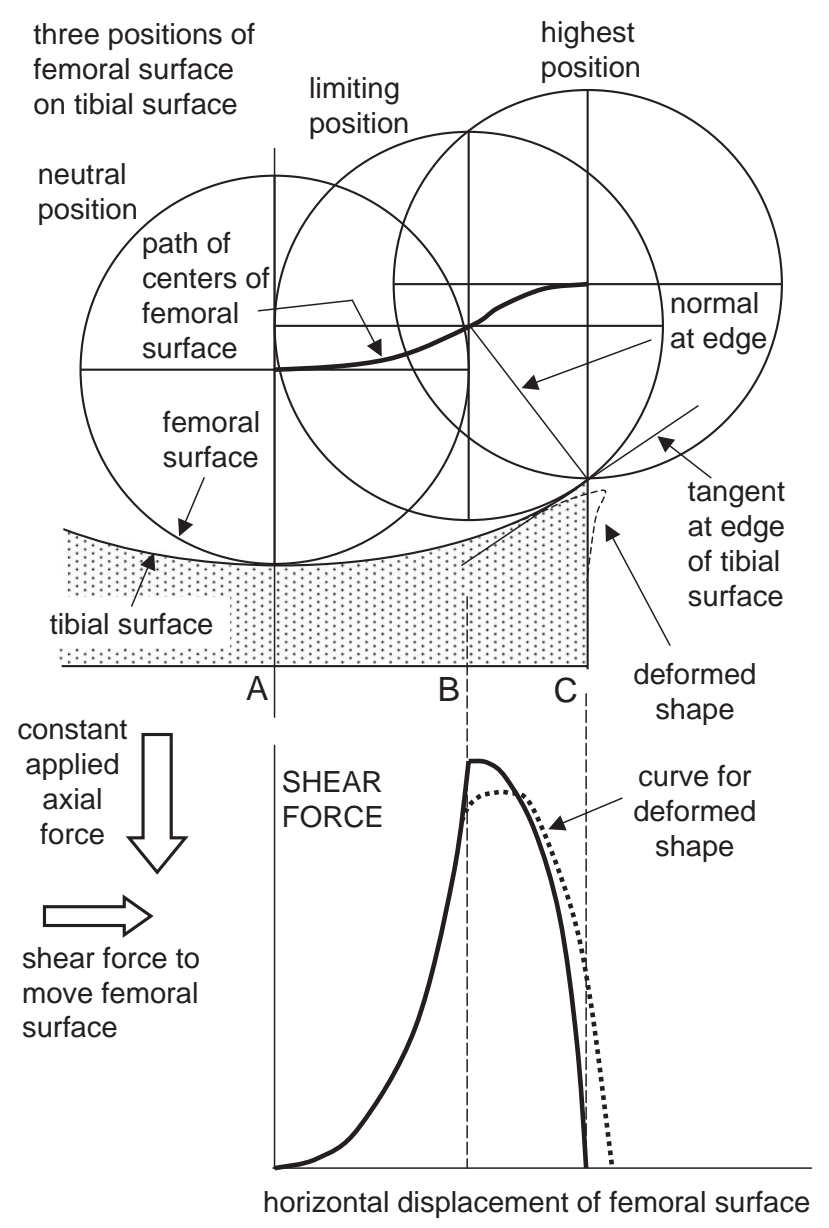

Fig. 2. The femur starts at the 'neutral position'. As the femur moves forward relative to the tibia (in the actual test the tibia moves; for the purposes of illustration, it is clearer to show the femur displacing) the shear force increases to a maximum when the 'limiting position' is reached. The force then reduces to zero at the 'highest position'. Deformation of the plastic at the edge modifies the curve.

different ways. For the AP test, the rotation was controlled to maintain zero rotation (as apposed to zero axial torque as described above). For the rotation test, the AP actuator was controlled to zero displacement (as apposed to zero AP force as described above). The rationale for these tests was that the bearing surfaces of some TKRs are asymmetric. In the sagittal view, the radii of both the femoral and tibial surfaces can change around the curve. Hence, controlling either displacement or force should result in different constraint results.

Having completed the above tests at the first angle of flexion, the femoral component was sent at the next angle and the test repeated.

In this paper, only the results for three different TKRs are shown at zero degrees flexion, axial force of $712 \mathrm{~N}$ ( 1 body weight), the AP test with the torque held to zero, and the rotation test with the AP force held to zero. 


\subsection{Details of the three implants}

The implants were new and standard sized, as supplied by the manufacturer. The Smith \& Nephew Genesis II, where the tibial component was set at a posterior slope of $3^{\circ}$; the Johnson \& Johnson PFC Sigma, where the tibial component was set at a posterior slope of $0^{\circ}$; the Zimmer Nexgen CR, where the tibial slope was set at $7^{\circ}$.

\subsection{Details of the AP test}

Flexion angle $0^{\circ}$, axial torque held constant at zero velocity of AP motion $1.0 \mathrm{~mm} / \mathrm{s}$, freedom of ML motion and varus-valgus rotation (allowing the components to move in these degrees of freedom if their geometry dictated).

\subsection{Details of the rotation test}

Flexion angle $0^{\circ}$, AP force held constant at zero, rate of angular rotation $1.0^{\circ} / \mathrm{s}$, freedom of ML motion and varus-valgus rotation (allowing the components to move in these degrees of freedom if their geometry dictated).

\section{Results}

\subsection{Repeatability}

The preparatory tests were carried using distilled water for convenience, while the actual test runs used calf serum in order to conform with the standards used in wear testing. However, it was found that the curves with the two lubricants were virtually identical, implying that the coefficients of friction were similar. It would not be expected that the lubricant would affect deformation or any other factor in these tests. It is concluded that distilled water can be used for these tests, which is simpler and more convenient to use than serum.

The test to determine the neutral position of the femur on the tibia with the application of an axial compressive force, gave accurate and repeatable results. By choosing the appropriate scale for the height vs horizontal displacement curve, a distinct U-shape was observed. On the other hand, the test to determine the limits of displacement and rotation required careful control. From Fig. 2, if the surfaces were rigid bodies, a distinct maximum would be reached in the shear force versus horizontal displacement curve. However, due to deformation at the edges of the plastic, and the timedependent visco-elastic behaviour of this deformation, a distinct maximum was not observed. Rather, the curve was rounded, as shown in Fig. 2. Moreover, in successive runs, the deformation could progress such that the curve fell off, bringing the femoral component into the zone between the 'limiting position' and the 'highest position'. Hence, having found the limits, it would be preferable to back off by say $0.5 \mathrm{~mm}$ and $0.5^{\circ}$ for the actual test runs.

The two sets of three curves that were obtained for each test (see above under General Test Method) were virtually identical, so it was concluded that the first set of three runs was satisfactory.

\subsection{AP test}

The AP force versus AP displacement curves for the three TKR designs are shown in Fig. 3. It is useful to compare the shapes of the curves to those of Fig. 2, at least for the upslope part of the curves. When the tibia was displaced anterior, all three curves reached a force limit and then the force began to reduce. After this point, the direction of motion of the tibial component was reversed. The displacement at the peak forces can be regarded (Fig. 2) as occurring at the limiting position of the femur on the tibia. For the three designs, these limiting values are approximately $2.5 \mathrm{~mm}$ for the Genesis, $4 \mathrm{~mm}$ for the NexGen, and $6 \mathrm{~mm}$ for the Sigma. In the opposite direction, the values are respectively 9 , 16 and $8 \mathrm{~mm}$. It is noted that AP force values to reach these maxima, and consequently the stiffnesses, differ significantly between the three designs. All of the curves show a hysteresis amounting to approximately $85 \mathrm{~N}$. Based on Luger et al. (1997), we attribute this primarily to metal-plastic friction, with a coefficient of friction of $\frac{42.5}{712}=0.06$.

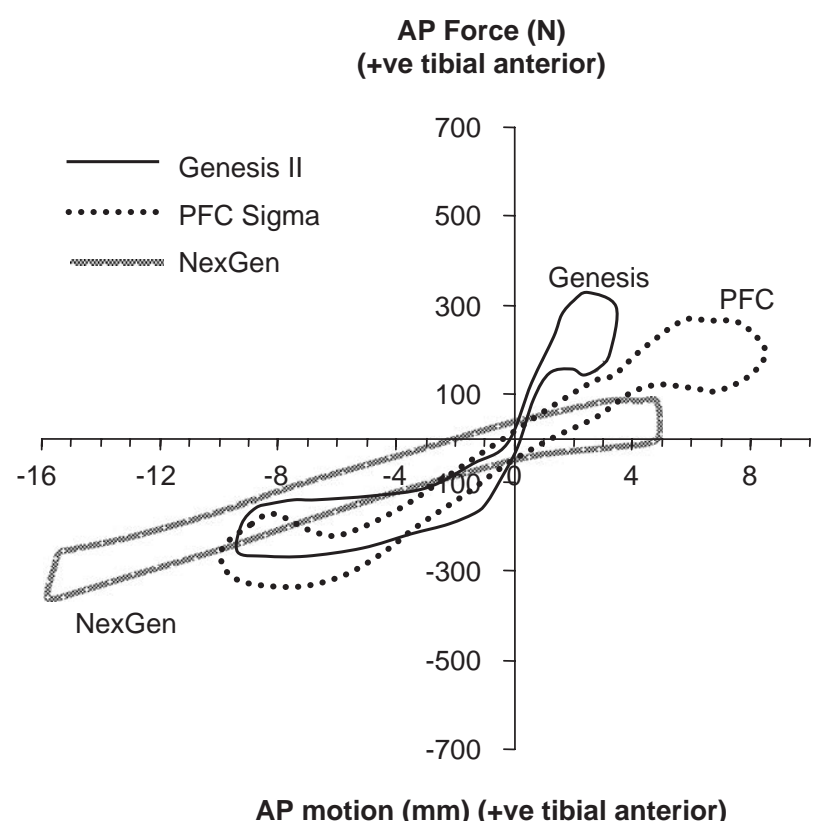

Fig. 3. The AP test for the 3 TKRs. The AP force is plotted against AP displacement (horizontal axis). All of the curves have just passed the 'limiting position'. 


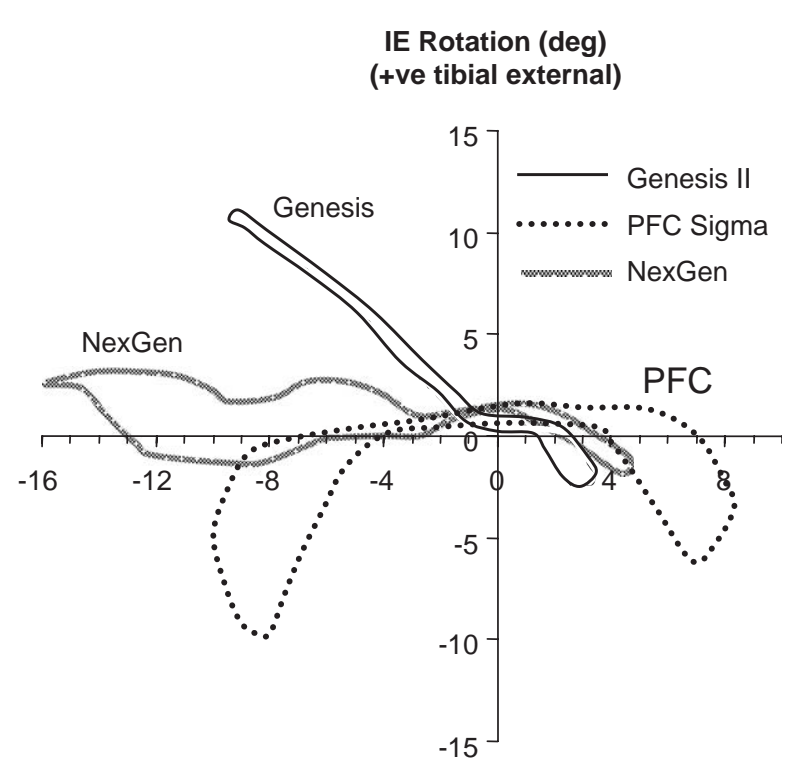

AP motion (mm) (+ve tibial anterior)

Fig. 4. The internal-external rotation occurring during the AP test.

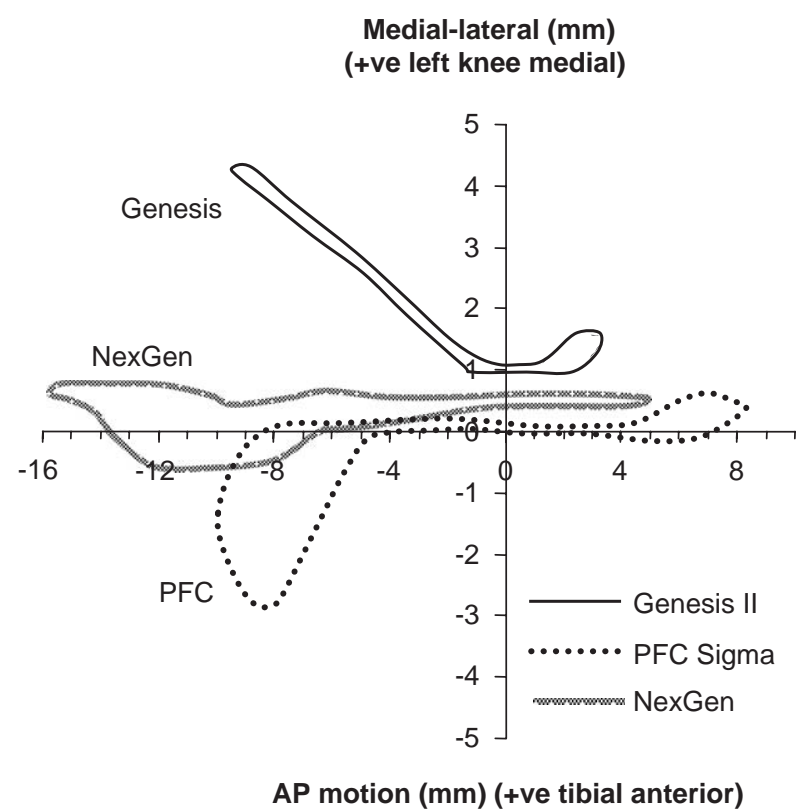

Fig. 5. The ML displacement occurring during the AP test.

During this AP test, where the axial torque was held to zero, the rotation versus AP displacement was recorded (Fig. 4). Large rotations of up to $10^{\circ}$ were recorded for the Genesis II and PFC Sigma. The rotations for the NexGen were only $1-2^{\circ}$.

The ML displacements for the Genesis II and PFC Sigma were 4 and $3 \mathrm{~mm}$, respectively (Fig. 5), while the varus rotations for the same two designs were $4^{\circ}$ (Fig. 6).

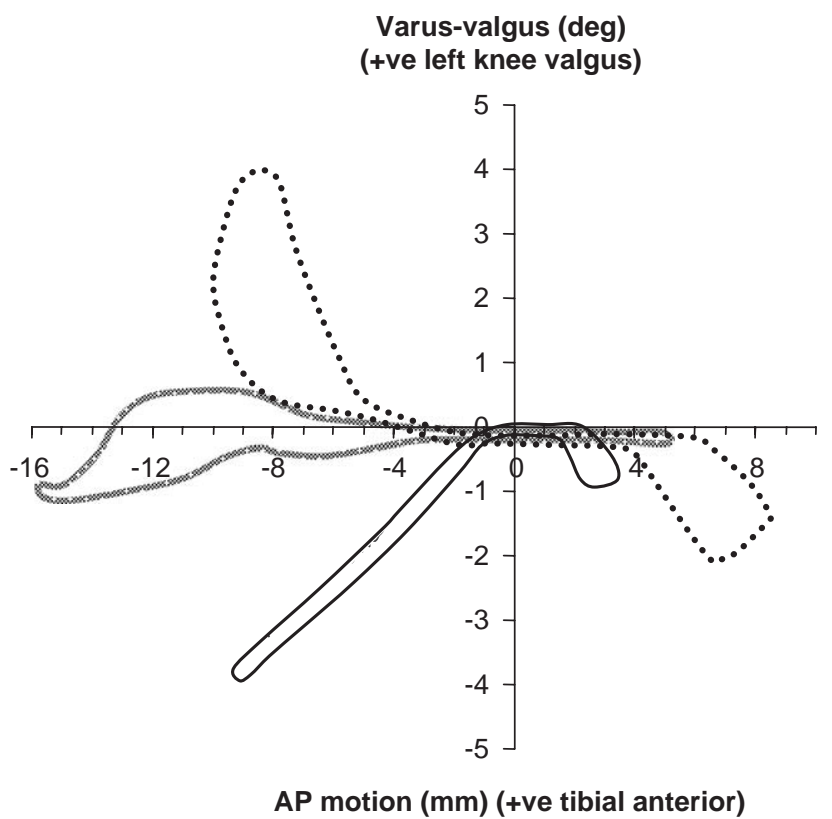

Fig. 6. The varus-valgus rotation occurring during the AP test.

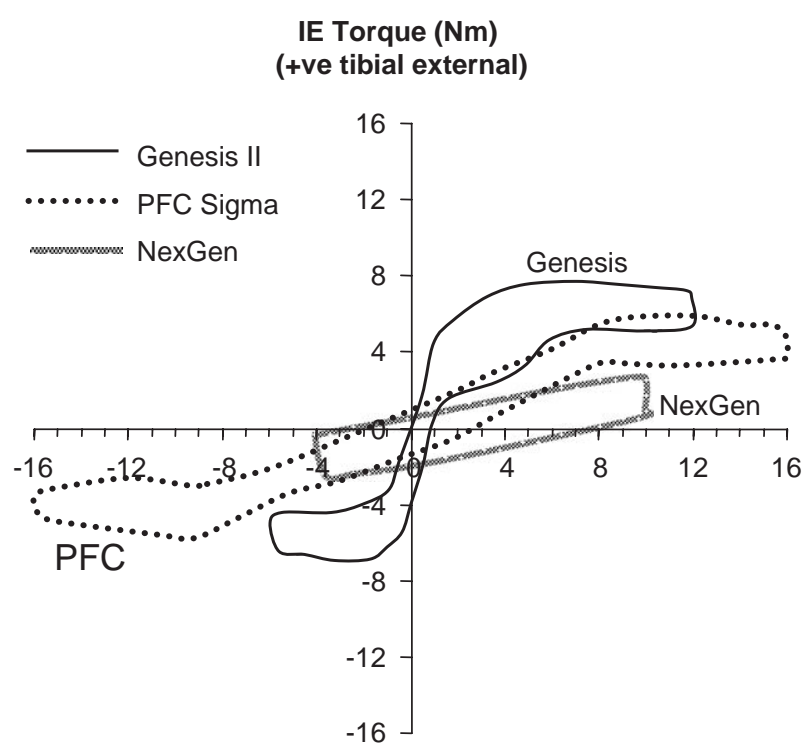

IE Rotation Angle (deg) (+ve tibial external)

Fig. 7. The rotation test for the 3 TKRs. The internal-external rotation is plotted against the rotation angle.

\subsection{Internal-external rotation test}

The general trends for the AP test were repeated for the rotation test. Fig. 7 shows the torque versus rotation curves. It was difficult to identify the rotation values at the peak torques because of the extended flat regions where the rotation increased with little change in torque. However, the relative stiffnesses of the three designs about the neutral position were similar to those in the 


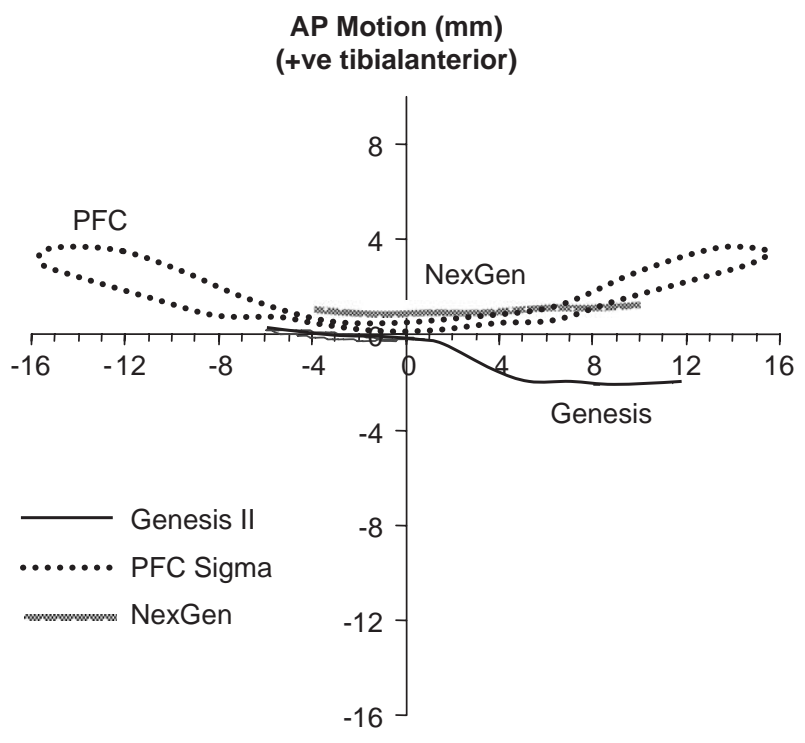

IE Rotation Angle (deg) (+ve tibial external)

Fig. 8. The AP displacement occurring during the rotation test.

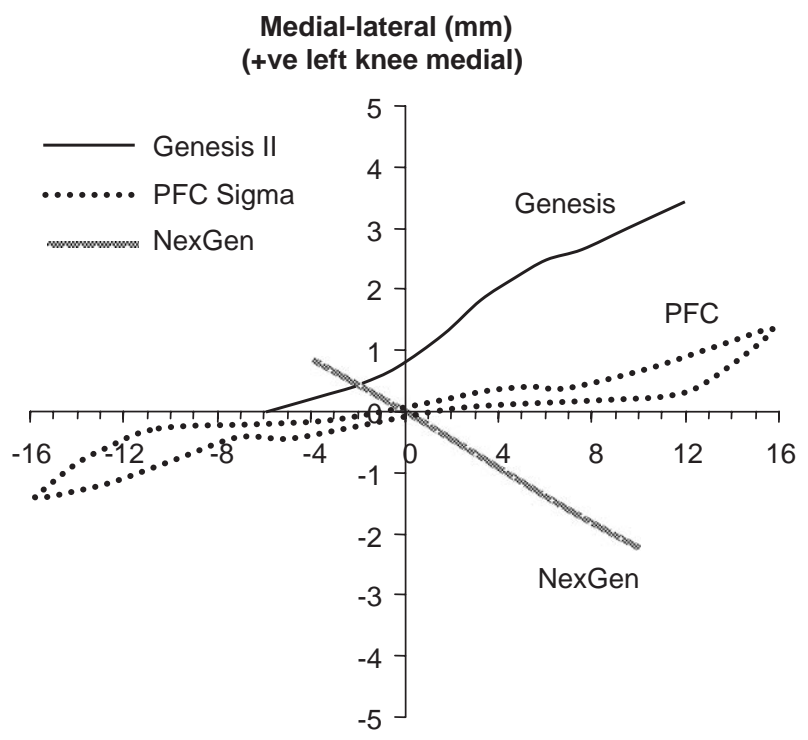

IE Rotation Angle (deg) (+ve tibial external)

Fig. 9. The ML displacement occurring during the rotation test.

AP test. During the tests, the AP motions of the Genesis and Sigma were 3-4 mm (Fig. 8). The ML displacements were up to $3 \mathrm{~mm}$ (Fig. 9), while the varus-valgus rotations were approximately $2.5^{\circ}$ (Fig. 9).

\section{Discussion}

The test machine used was an advanced multi-axis type of MTS which could control and record the forces and displacements along the vertical and AP axes, and the torques and rotations about the vertical axis. In addition, the special fixture allowed freedom of motion along the ML-axis (the displacement being measured by an added transducer) and about the AP-axis (the rotation being measured by an added transducer), and the data logging synchronized all together through the MTS machine controller. This configuration facilitated tests which would more resemble the conditions occurring in vivo where in general, degrees of freedom are not locked by mechanical means but are either allowed to occur with soft tissues restraining motions at the extremes, or where superimposed muscle actions act to modify the effect of the external forces.

The set-up allowed sufficient variable control to isolate those constraint effects inherent within the TKR design, from in vivo variables such as the soft tissue contribution, patient variability, and surgical alignment. Carrying out simple AP tests for example using this equipment, revealed that there were significant motions which simultaneously occurred along and about other axes. This provided a more complete understanding as to how the TKRs might function in vivo, as well as how the tests themselves might be carried out more realistically.

The test results shown are only a small sample of the complete data collected. We chose to show only $0^{\circ}$ flexion because it is an important angle in function, and striking differences were found between the three TKRs. The stiffnesses of the curves passing through zero (Eq. (2)), and the total stiffnesses (Eq. (1)) for both AP and rotation were dramatically different. The magnitudes of the displacements and rotations were also strikingly different. The actual maximum values of the shear force (approximately $300 \mathrm{~N}$ ) and the torque (approximately $8 \mathrm{Nm}$ ) were within the limits of normal function. However, in tests carried out at $1428 \mathrm{~N}$ $(2 \times$ body weight $)$ axial compressive load, the maximum values would not likely be realized in function except in extreme conditions.

The motions measured in the other degrees of freedom during the AP and rotation tests were significant. For example in AP, up to $10^{\circ}$ in internalexternal rotation, $4^{\circ}$ in varus-valgus, and $4 \mathrm{~mm}$ in $\mathrm{ML}$ displacement occurred (Fig. 10). Clearly if these degrees of freedom had been locked instead of being free, the TKRs would have been prevented from moving in their natural paths. Furthermore, the motions themselves would have been abnormal. For example in internalexternal rotation, if AP displacement was locked, on rotation one condyle would be in contact with the plastic while the other would be not in contact at all. Hence, even if the rotations and displacements in the other degrees of freedom were not measured, they have to be free, requiring appropriate test fixture design, such as low-friction linear slides. It is interesting to speculate whether an asymmetric knee would experience rotation in function when an AP shear force was acting. 


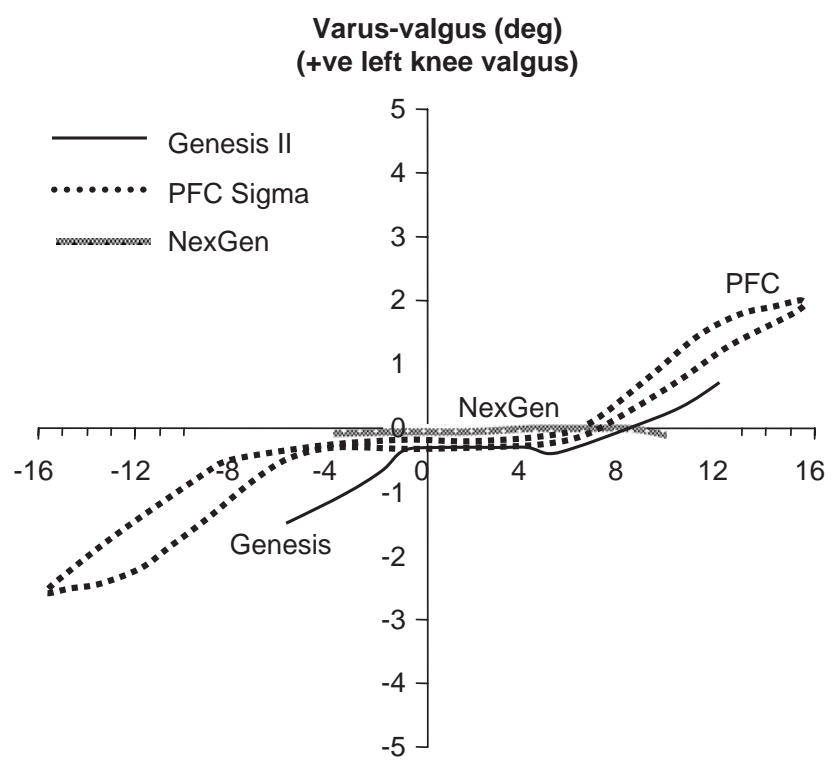

IE Rotation Angle (deg) (+ve tibial external)

Fig. 10. The varus-valgus rotation occurring during the rotation test.

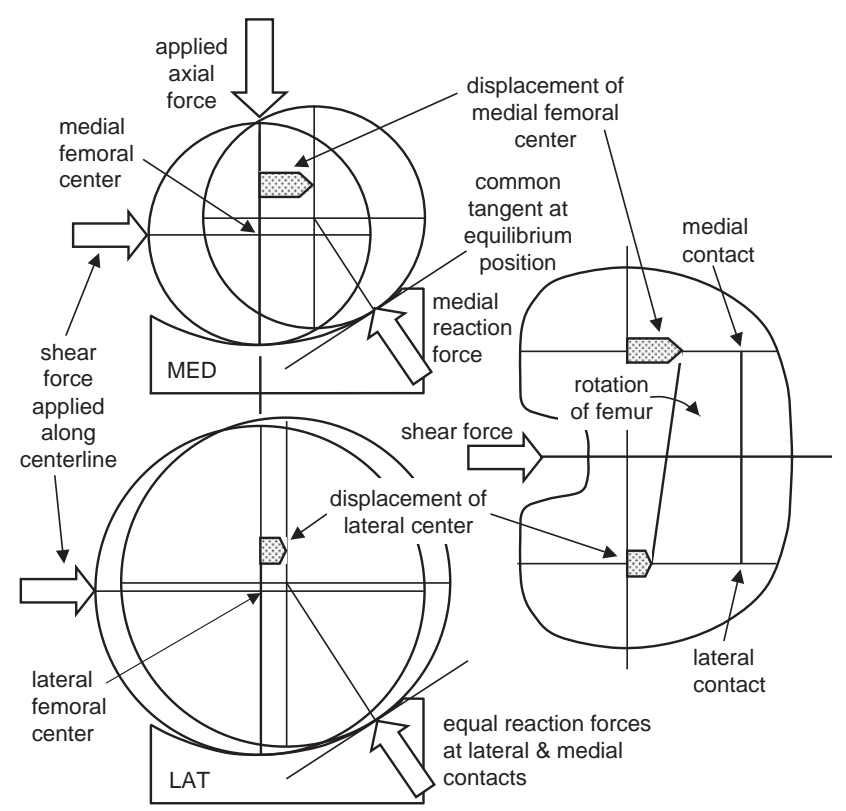

Fig. 11. The behaviour of an asymmetric femoral component, with smaller medial radius and larger lateral radius, when an AP force is applied along the center-line. The medial condyle displaces further anteriorly than the lateral, resulting in an axial rotation. However, the contact points are exactly in line.

An explanation for the occurrence of the other motions is presented in Fig. 11, which depicts an AP test. The tibial surfaces are the same on the medial and lateral sides, but the femoral condyles are asymmetric. This was the case with the Genesis II and PFC Sigma designs. It is noted that the NexGen has asymmetric femoral condyles but this had no effect at $0^{\circ}$ in the AP test. The figure shows a smaller medial radius and a larger lateral radius. For an AP force applied centrally, the horizontal component of the reaction forces at each condyle must be the same. Therefore the common tangents at the contacts must have the same slope. The medial condyle displaces horizontally more than the lateral. Therefore the femoral component must rotate about a vertical axis (internal-external rotation). It is very interesting to observe that while the reference axis of the femur rotates, the line joining the actual contact points remains parallel. Hence in any depiction of such tests (whether on a test machine, or in fluoroscopy studies for example) care must be taken to define whether reference axes or contact points are being mapped. Also from Fig. 11 it can be seen that the vertical displacement of the medial side is greater than that of the lateral, indicating that valgus rotation occurred.

If TKRs with symmetric femoral and tibial surfaces had been tested, the motions in the other degrees of freedom would have been zero in an AP test. (Even then, manufacturing tolerances would produce inequalities of forces between medial and lateral.) However, it is interesting to note that in a rotation test, even with perfect manufacturing, the pure rotation torque would in itself cause some AP motion on most implant designs. The reason is that the radius of the femoral condyles in the sagittal plane is usually not constant, being larger distally and anteriorly than posteriorly. This would result in AP and ML displacements on rotation. The higher radius of curvature on the anterior side of one condyle would reach the anterior slope of the plastic and start pushing forward the tibial component at a different time than the smaller radius of curvature of the posterior of the other femoral condyle reaching the posterior slope of the plastic. All of these considerations reinforce the conclusion that other degrees of freedom as noted above need to be free.

Can a standard test be recommended from our study? To answer this, the objectives must be defined. One objective is to understand the behaviour of the TKR in vivo, in terms of the maximum displacements and rotations the bearing surfaces are capable of, the likely displacements and rotations that would occur in function, the feeling of stability experienced by the patient, and to what extent ligaments and muscular control would be necessary to control motion. In this case, tests such as we have shown here would be required. The output numerical data would be the limits of AP and rotation, and the maximum forces and torques at these limits. This data would be shown at different angles of flexion, notably $0^{\circ}, 20^{\circ}$ (stance phase of walking), $90^{\circ}$ (climbing steep steps, rising from a chair) and the maximum flexion the knee is capable of. Another objective is to characterize in a simple way the constraint of a TKR in the two principle modes of 
motion, AP and rotation. In this case, all that is required is the stiffness, defined by Eqs. (1) or (2), of the curves before the limiting position (Fig. 2) for the angles of flexion specified above. Going further, for a range of knees, ranges of low, medium or high stiffness could be defined.

Our tests were restricted to fixed-bearing knees with partially conforming bearing surfaces. If the tibial bearing surfaces were flat in AP, the AP force would be equal to the frictional force until the limiting position was reached. The same would apply for unrestricted rotation, as in a rotating platform mobile bearing design. A design with a stop however, such as a PS type, or where a mobile bearing has limits, the applied force on impact would increase excessively. In this case, a displacement or rotation limit would be needed, or a maximum safe force or torque.

In conclusion, using a sophisticated test machine, AP and rotational constraint tests were carried out on three widely used fixed-bearing TKRs. The results showed that for such testing to be meaningful, degrees of freedom other than those being tested must be free. If not, anomalous results would be obtained. For sufficient characterization of a TKR, there are many conditions under which the tests need to be carried out. However, a single axial force is sufficient, and distilled water can be used, but a range of flexion angles must be tested. The presentation of the data can be holistic or simplified, depending on the purpose of the testing.

\section{Acknowledgements}

We thank the manufacturers (Smith \& Nephew, De-Puy Johnson \& Johnson, and Zimmer) for supplying the samples. Technical assistance was provided by Richard Croson, Dana Hess and Joel Wisenberger.

\section{References}

Black, J.D., Greenwald, A.S., 1979. Transactions of Orthopaedic Research Society 4, 96.

Black, J.D., Matejczyk, M.B., Greenwald, A.S., Wilde, A.H., 1977. Rotational characteristics of non-hinged total knee replacements. Transactions of Orthopaedic Research Society 2, 56.

Heim, C.S., Postak, P.D., Plaxton, N.A., Greenwald, A.S., 2001. Classification of mobile-bearing knee designs: mobility and constraint. Journal of Bone and Joint Surgery 83-A, S2-32.

Luger, E., Sathasivam, S., Walker, P.S., 1997. Inherent differences in the laxity and stability between the intact knee and total knee replacements. The Knee 4, 7-14.

Thatcher, J.C., Zhou, X-M., Walker, P.S., 1987. Inherent laxity in total knee prostheses. Journal of Arthroplasty 2, 199-207.

Werner, F., Foster, D., Murray, C.G., 1978. The influence of design on the transmission of torque across knee prostheses. Journal of Bone and Joint Surgery 60-A, 342-348. 